\title{
The antimicrobial efficacy of 'MGP' gutta-percha in vitro
}

\author{
A. L. Shur ${ }^{1}$, C. M. Sedgley ${ }^{1}$ \& J. C. Fenno ${ }^{2}$ \\ Departments of ${ }^{1}$ Cariology, Restorative Sciences and Endodontics, and ${ }^{2}$ Biologic and Material Sciences, University of Michigan \\ School of Dentistry, Ann Arbor, MI, USA
}

\begin{abstract}
Shur AL, Sedgley CM, Fenno JC. The antimicrobial efficacy of 'MGP' gutta-percha in vitro. International Endodontic Journal, 36, 616-621, 2003.
\end{abstract}

Aim To determine whether 'MGP' gutta-percha (Westport, CT, USA), a commercially available gutta-percha containing iodoform, inhibits the growth of potential endodontic pathogens.

Methodology Inocula of Enterococcus faecalis, Staphylococcus aureus, Escherichia coli, Pseudomonas aeruginosa, Streptococcus sanguis, Fusobacterium nucleatum and Actinomyces odontolyticus were spread onto the surface of agar plates. 'MGP' gutta-percha cones presoaked in sterile water were transferred to the inoculated agar and incubated at $37^{\circ} \mathrm{C}$ aerobically or anaerobically as required for optimal growth. Identical studies were performed using iodoform-free gutta-percha and sterile

\section{Introduction}

Bacteria are crucial to the development of pulpal and periradicular disease (Kakehashi et al. 1966, Sundqvist 1976). The bacterial flora associated with infected pulps is polymicrobial and predominantly anaerobic (Sundqvist 1976, Siqueira et al. 2000, Rolph et al. 2001), whereas the flora in teeth with persisting periapical lesions contains fewer species and proportionally less anaerobes (Sundqvist et al. 1998). Whilst Enterococci usually make up a small proportion of the initial flora in the untreated root canal (Sundqvist 1992, Sjögren et al. 1997, Siqueira et al. 2002), they are the species most commonly recov-

Correspondence: Dr Christine Sedgley, Department of Cariology, Restorative Sciences and Endodontics, University of Michigan Dental School, 1011 N. University Drive, Ann Arbor, MI 48109-1078, USA (Tel.: +1 734 6474182; fax: +1 734 9361597; e-mail: csedgley@umich.edu). paper disks saturated with $10 \%$ povidone-iodine. Following incubation, zones of inhibition around the 'MGP' gutta-percha, iodoform-free gutta-percha and disks were evaluated.

Results Povidone-iodine inhibited all the strains. Iodoform-free gutta-percha inhibited S. sanguis and A. odontolyticus. 'MGP' gutta-percha inhibited S. aureus, S. sanguis, A. odontolyticus and F. nucleatum. Neither iodoform-free gutta-percha nor 'MGP' gutta-percha inhibited growth of E. faecalis, E. coli or P. aeruginosa.

Conclusions Compared to iodoform-free guttapercha, iodoform-containing 'MGP' gutta-percha had an inhibitory effect in vitro on $S$. aureus and $F$. nucleatum, but not on E. faecalis, E. coli or P. aeruginosa.

Keywords: antimicrobial, gutta-percha, in vitro, iodoform, 'MGP' gutta-percha.

Received 2 December 2002; accepted 8 May 2003 ered from root canals of teeth with failed root treatment (Molander et al. 1998, Sundqvist et al. 1998, Hancock et al. 2001) and have been implicated in persistent root canal infections (Haapasalo et al.1983, Byström \& Sundqvist 1985). Ideally, all bacteria should be eradicated prior to obturation; however, bacteria may persist in the root canal system despite debridement and disinfection (Haapasalo \& Örstavik 1987, Nair et al. 1990, Sjögren et al. 1997, Sundqvist et al. 1998). Recent data indicate that $5 \%$ iodine potassium iodide might reduce the frequency of persisting Enterococci in the root canal system (Molander et al. 1999, Peciuliene et al. 2001).

Grossman (1940) advocated that an ideal root canal filling material should be bacteriostatic. Whilst the zinc oxide in gutta-percha cones might impart antimicrobial activity (Moorer\&Genet1982a), untilrecently, contemporary gutta-percha cones were neither intentionally antiseptic nor bacteriostatic. Recently, iodoform has been included in gutta-percha cones (Lone Star Technologies, 
Westport, CT, USA); this product is marketed as 'MGP' gutta-percha and has US Food and Drug Administration (FDA) approval. The developers state that 'the iodoform in the MGP cones remains inert until it comes in contact with tissue fluids that activate the free iodine' (Martin \& Martin 1999). In vitro investigations by the developers showed 'MGP' gutta-percha to have antimicrobial activity against Streptococcus viridans, Staphylococcus aureus and Bacteroides fragilis (Martin \& Martin 1999).

To date, minimal data are available on iodoform-containing 'MGP' gutta-percha points. By observing zones of inhibition, 'MGP' gutta-percha had antimicrobial activity against Streptococcus sanguis, but not against Enterococcus faecalis (Silver et al. 2000). Preliminary reports (abstracts only) indicated that 'MGP' guttapercha was ineffective against E. faecalis in an in vitro leakage study (Bruchmiller et al. 2000), and had no antimicrobial activity against bacteria from human oral plaque samples (Dryden et al. 2000). The aims of the present in vitro investigation were to independently assess the developers' findings and to determine, using the developers' methodology, whether gutta-percha containing 10\% iodoform ('MGP' gutta-percha) inhibited the growth of aerobic and anaerobic bacterial species associated with endodontic infections.

\section{Materials and methods}

\section{Microorganisms}

The eight bacterial strains selected for study are listed in Table 1. S. aureus was obtained from the American Type Culture Collection (Manassas, VA, USA). All other bacteria were recovered from strain stocks stored at $-80{ }^{\circ} \mathrm{C}$ in the University of Michigan School of Dentistry, Ann Arbor, MI, USA.

\section{Antimicrobial experiments}

E. faecalis, $S$. aureus, $P$. aeruginosa and E. coli were grown in $5 \mathrm{~mL}$ of Todd Hewitt Broth (THB; Difco, Detroit, MI,
USA) and incubated for $24 \mathrm{~h}$ at $37{ }^{\circ} \mathrm{C}$ aerobically. After $24 \mathrm{~h}$, each sample was diluted $1: 10$ in $\mathrm{THB}$, and $50 \mu \mathrm{L}$ were spread onto the surface of Trypticase Soy Agar (TSA; Difco, Detroit, MI, USA) using a sterile glass spreader.

The developers stated that the iodoform within the 'MGP' gutta-percha cones remains inert until it comes in contact with tissue fluids, which then activate the free iodine; they used a 'leakage agent' in their study to release the free iodine (Martin \& Martin 1999). Adopting the developers' methods, the 'MGP' gutta-percha cones were 'soaked' by complete immersion in $2 \mathrm{~mL}$ of sterile water in a test tube for $1 \mathrm{~h}$ (Martin \& Martin 1999). Control gutta-percha points without iodoform (Hygenic Corp., Akron, OH, USA) were similarly treated. Following soaking, cones were transferred to inoculated agar plates as described below. For each agar plate, a set of five cones were individually soaked.

Four TSA plates were used for each strain and treated as follows:

- Plate 1: Five 'MGP' gutta-percha points (size 30) were aseptically transferred to the centre of each previously inoculated plate.

- Plate 2: Five gutta-percha points (size 30) without iodoform were aseptically transferred to the centre of each previously inoculated plate.

- Plate 3: A sterile paper disk, $7 \mathrm{~mm}$ in diameter, saturated with $20 \mu \mathrm{L}$ of $10 \%$ povidone-iodine (Betadine, Purdue Frederick Co., Norwalk, CT, USA) was aseptically transferred to the centre of each previously inoculated plate.

- Plate 4: This plate received no other treatment and served as a positive control for growth of the microorganisms.

All plates were incubated for $24 \mathrm{~h}$ at $37^{\circ} \mathrm{C}$ aerobically. For each strain, experiments were performed in triplicate.

For experiments using strains grown under anaerobic conditions, the procedures were identical to the above with the following variations:
Table 1 Antibacterial effects of 'MGP' gutta-percha, regular gutta-percha and povidone-iodine disks

\begin{tabular}{llll}
\hline Bacterial strains & 'MGP' gutta-percha & Regular gutta-percha & Povidone-iodine \\
\hline E. faecalis (OGI) & - & - & + \\
E. faecalis (ATCC 47077) & - & - & + \\
S. aureus (ATCC 6538) & + & - & + \\
P. aeruginosa (UME) & - & - & + \\
E. coli (SM10 pir) & - & - & + \\
S. sanguis (ATCC 10556) & + & + & + \\
F. nucleatum (ATCC 25586) & + & - & + \\
A. odontolyticus (ATCC 17982) & + & + & + \\
\hline +, antimicrobial; -, not antimicrobial. &
\end{tabular}




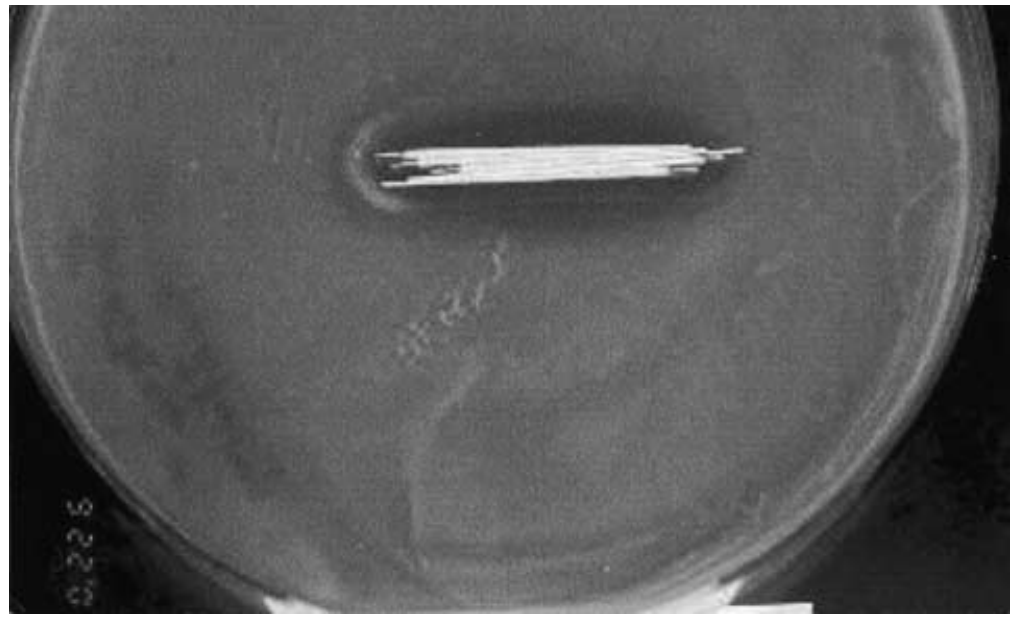

Figure 1 Inhibition of growth by 'MGP' gutta-percha.
1 Microorganisms were grown in Schadler broth (Difco, Detroit, MI, USA).

2 S. sanguis was incubated for 24 h and $A$. odontolyticus and F. nucleatum were incubated for $48 \mathrm{~h}$ at $37^{\circ} \mathrm{C}$ in an anaerobic culture chamber.

3 Anaerobes were spread on blood agar plates (Anaerobe Systems, Morgan Hills, CA, USA).

4 Plates were incubated for $24-48 \mathrm{~h}$ in an anaerobic culture chamber at $37{ }^{\circ} \mathrm{C}$.

After incubation, all the plates were visually inspected by two examiners for evidence of growth on the control plates and inhibition of bacterial growth on the other plates. Absence of microbial growth immediately adjacent to the 'MGP' gutta-percha, regular gutta-percha and iodine disks indicated antimicrobial activity.

\section{Results}

Uniform growth was evident on all control plates. Results for all other plates are presented in Table 1. Samples from triplicate trials yielded consistent results.

Povidone-iodine inhibited all strains of bacteria tested. Gutta-percha without iodoform inhibited S. sanguis and A. odontolyticus only. 'MGP' gutta-percha inhibited the growth of $S$. sanguis, A. odontolyticus, F. nucleatum and S. aureus (Fig. 1), but not E. faecalis, P. aeruginosa and E. coli (Fig. 2).

\section{Discussion}

In the absence of a coronal seal, $50 \%$ of teeth obturated with gutta-percha and sealer and exposed to

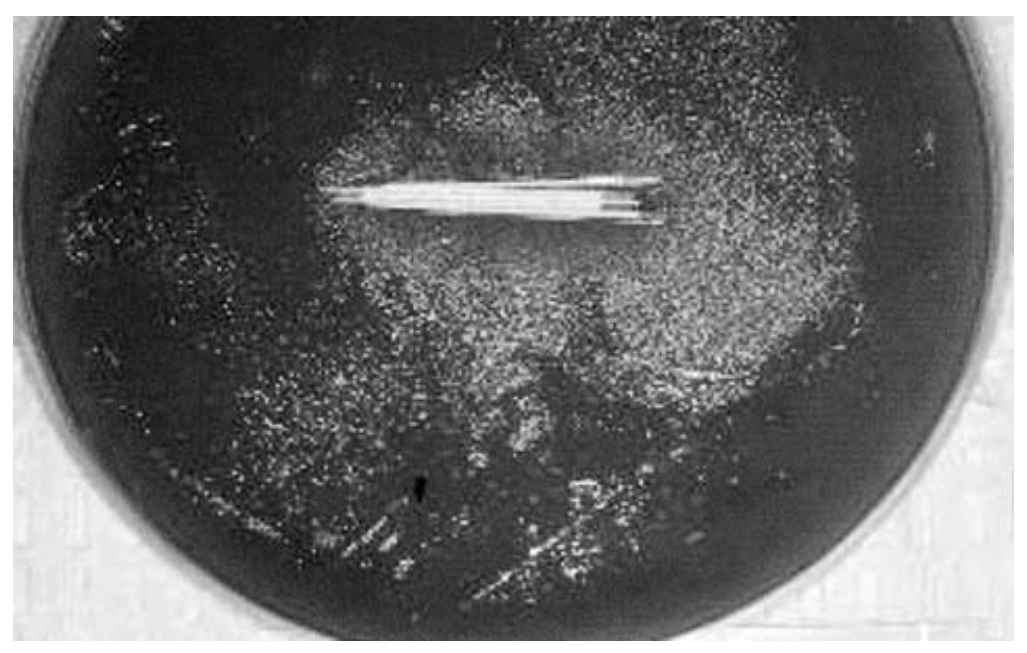

Figure 2 No inhibition of growth by 'MGP'gutta-percha. 
Staphylococcus epidermidis and Proteus vulgaris had complete bacterial penetration throughout the entire length of the root canal within 19 days (Torabinejad et al. 1990). As root canals may not be completely filled, regardless of obturation technique (Eguchi et al. 1985), and sealer may be lost over time (Peters 1986), theoretically, the prognosis of endodontic treatment could be enhanced if the obturation material was able to prevent bacterial recontamination.

'MGP' gutta-percha contains $10 \%$ iodoform $\left(\mathrm{CHI}_{3}\right)$, a crystalline substance, which is soluble in chloroform and ether but has low solubility in water (Budavari 1989). Iodophors, for example, povidone-iodine or 'Betadine', are iodine compounds linked with surfaceactive agents; these compounds interact with cell walls of microorganisms causing pore formation or generating solid-liquid interfaces at the lipid membrane level, which lead to loss of cytosol material and enzyme denaturation (Fleischer \& Reimer 1997, Schreier et al. 1997). The product information provided by the manufacturer (Lone Star Technologies, Westport, CT, USA) states that the iodoform in the MGP cones is inert until it comes into contact with tissue fluids, which activate the free iodine', and make it available to inhibit bacteria remaining in the canal, or which enter the canal via leakage. Whilst an ideal root-filling material should be bacteriostatic (Grossman 1940), one could speculate that in the clinical situation, the presence of a heavy microbial load, as well as tissue fluids, would dilute the iodine concentration, thereby diminishing the antibacterial effect. Sealer solubility and thickness, shown to influence fluid leakage (Wu et al. 1995), would also be expected to moderate access between the medication and microorganisms. Further, given the low solubility of iodoform in water (Budavari 1989), both in vitro and in vivo quantitative investigations on the release of free iodine from 'MGP' gutta-percha over an extended and clinically relevant time period are indicated.

In the present study, $10 \%$ povidone-iodine inhibited all eight bacterial strains examined, whilst the growth of $S$. aureus and F. nucleatum only was inhibited by 'MGP' gutta-percha compared to iodoform-free guttapercha. Similarly, chlorhexidine- and calcium hydroxide-impregnated gutta-percha were less bactericidal than the disinfectants alone (Barthel et al. 2002). Whilst the final volume and concentration of free iodine released from the 'MGP' gutta-percha may have differed from that released from paper disks saturated with povidone-iodine, the present results suggest that the release and activation of antimicrobial free iodine from
'MGP' gutta-percha, as claimed by the developers, is not substantial. Based on the present in vitro results, reliance on the antimicrobial activity of free iodine released from 'MGP'gutta-percha to disinfect infected root canals cannot be supported.

Iodoform-free gutta-percha cones were used as negative controls; other differences between the iodoformfree gutta-percha and 'MGP'gutta-percha were not investigated but may exist. Interestingly, the growth of two strains, S. sanguis and A. odontolyticus, was inhibited by iodoform-free gutta-percha. Similarly, traditional gutta-percha cones have been shown to inhibit several bacterial species in vitro (Moorer \& Genet 1982b, Attin et al. 2001), perhaps, in part, because of the inherent antibacterial activity of zinc oxide within the gutta-percha (Moorer \& Genet 1982a).

The present data on $S$. aureus correlated with those of the developers. However, none of the studies thus far on 'MGP'gutta-percha using this methodology have adequately quantified zones of inhibition around the gutta-percha. Whilst the clusters of five cones in close proximity (Figs 1 and 2) used in this and the developers' studies may simulate the number of cones used during lateral condensation procedures in vivo, the suitability of 'MGP' gutta-percha for 'any obturation technique' (Martin \& Martin 1999) has yet to be confirmed. In view of the $120{ }^{\circ} \mathrm{C}$ melting point of iodoform (Budavari 1989), and in an effort to permit quantification, further studies could examine the effects, if any, of heat-activated obturation techniques on the antimicrobial characteristics of 'MGP' gutta-percha using gutta-percha disks of standardized diameter.

Finally, Sundqvist et al. (1998) reported that the most common isolate from teeth with failing endodontic therapy was E. faecalis. In this study, the growth of E. faecalis strains was not inhibited by either iodoform-free gutta-percha or 'MGP' gutta-percha in agreement with previous investigators (Silver et al. 2000). Duration of presoaking the 'MGP'gutta-percha appeared to be unimportant, as Silver et al. (2000) soaked the gutta-percha cones for only $1 \mathrm{~min}$, as opposed to $60 \mathrm{~min}$ for this and the developers' investigations. The rationale behind the selection of a $1-\mathrm{h}$ presoaking time to simulate the in vivo model is unclear, and the experimental model (Martin \& Martin 1999) needs further investigation.

\section{Conclusion}

Iodoform-free gutta-percha inhibited S. sanguis and A. odontolyticus, whilst 'MGP' gutta-percha inhibited 
the growth of $S$. aureus, $S$. sanguis, A. odontolyticus and F. nucleatum, but not E. faecalis, E. coli and P. aeruginosa. Thus, the addition of iodoform to gutta-percha only had an antimicrobial effect on $S$. aureus and F. nucleatum of the eight strains tested. Based on these in vitro results, reliance on the antimicrobial activity of free iodine released from 'MGP' gutta-percha to disinfect infected root canals cannot be supported.

\section{Acknowledgements}

The authors would like to thank Lone Star Technologies for supplying the 'MGP' gutta-percha, as well as the University of Michigan Graduate Research Fund for providing financial support for this investigation.

\section{References}

Attin T, Zirkel C, Pelz K (2001) Antibacterial properties of electron beam-sterilized gutta-percha cones. Journal of Endodontics 27, 172-4.

Barthel CR, Zimmer S, Zilliges S, Schiller R, Gobel UB, Roulet JF (2002) In situ antimicrobial effectiveness of chlorhexidine and calcium hydroxide: gel and paste versus gutta-percha points. Journal of Endodontics 28, 427-30.

Bruchmiller T, Glickman GN, Suchina J (2000) Comparative analysis of MGP and standard gutta-percha using a bacterial leakage model. Journal of Endodontics 27, 216(abstract).

Budavari S (1989) The Merck Index, 11th edn. New Jersey: Merck.

Byström A, Sundqvist G (1985) The antibacterial action of sodium hypochlorite and EDTA in 60 cases of endodontic therapy. International Endodontic Journal 18, 35-40.

Dryden MT, Kim D, Huang GT-J (2000) Anti-microbial effects of gutta-percha on oral plaque. Journal of Endodontics 27, 234(abstract).

Eguchi DS, Peters DD, Hollinger JO, Lorton L (1985) A comparison of the area of the canal space occupied by gutta-percha following four gutta-percha obturation techniques using Procosol sealer. Journal of Endodontics 11, 166-75.

Fleischer W, Reimer K (1997) Povidone-iodine in antisepsis state of the art. Dermatology 195, 3-9.

Grossman LI (1940) Root Canal Therapy. Philadelphia: Lea \& Febiger.

Haapasalo M, Örstavik D (1987) In vitro infection and disinfection of dentinal tubules. Journal of Dental Research $\mathbf{6 6}$, 1375-9.

Haapasalo M, Ranta H, Ranta KT (1983) Facultative gram-negative enteric rods in persistent periapical infections. Acta Odontologica Scandinavica 41, 19-22.

Hancock HH, III, Sigurdsson A, Trope M, Moiseiwitsch J (2001) Bacteria isolated after unsuccessful endodontic treatment in a North American population. Oral Surgery, Oral Medicine, Oral Pathology, Oral Radiology and Endodontics 91, 579-86.
Kakehashi S, Stanley HR, Fitzgerald RJ (1966) The effects of surgical exposures of dental pulps in germ-free and conventional laboratory rats. Journal of the Southern Californian Dental Association 34, 449-51.

Martin H, Martin TR (1999) Iodoform gutta-percha: MGP, a new endodontic paradigm. Dentistry Today 18, 76-81.

Molander A, Reit C, Dahlén G, Kvist T (1998) Microbiological status of root-filled teeth with apical periodontitis. International Endodontic Journal 31, 1-7.

Molander A, Reit C, Dahlén G (1999) The antimicrobial effect of calcium hydroxide in root canals pretreated with $5 \%$ iodine potassium iodide. Endodontics and Dental Traumatology 15, 205-9.

Moorer WR, Genet JM (1982a) Antibacterial activity of guttapercha cones attributed to the zinc oxide component. Oral Surgery, Oral Medicine and Oral Pathology 53, 508-17.

MoorerWR, GenetJM (1982b) Evidence for antibacterial activity of endodontic gutta-percha cones. Oral Surgery, Oral Medicine and Oral Pathology 53, 503-7.

Nair PN, Sjögren U, Krey G, Kahnberg KE, Sundqvist G (1990) Intraradicular bacteria and fungi in root-filled, asymptomatic human teeth with therapy-resistant periapical lesions: a long-term light and electron microscopic follow-up study. Journal of Endodontics 16, 580-8.

Peciuliene V, Reynaud AH, Balciuniene I, Haapasalo M (2001) Isolation of yeasts and enteric bacteria in root-filled teeth with chronic apical periodontitis. International Endodontic Journal 34, 429-34.

Peters DD (1986) Two-year in vitro solubility evaluation of four gutta-percha sealer obturation techniques. Journal of Endodontics 12, 139-45.

Rolph HJ, Lennon A, Riggio MPet al. (2001) Molecular identification of microorganisms from endodontic infections. Journal of Clinical Microbiology 39, 3282-9.

Schreier H, Erdos G, Reimer K, Konig B, Konig W, Fleischer W (1997) Molecular effects of povidone-iodine on relevant microorganisms: an electron-microscopic and biochemical study. Dermatology 195, 111-6.

Silver GK, Taylor TL, Simons JHS (2000) Antimicrobial effect of iodoform gutta-percha cones on Streptococcus sanguis and Enterococcus faecalis. New Zealand Endodontic Journal 26, 1-3.

SiqueiraJF, Jr, Rocas IN, Souto R, de Uzeda M, Colombo AP (2000) Checkerboard DNA-DNA hybridization analysis of endodontic infections. Oral Surgery, Oral Medicine, Oral Pathology, Oral Radiology and Endodontics 89, 744-8.

SiqueiraJF, Jr, Rocas IN, Souto R, de Uzeda M, ColomboAP (2002) Actinomyces species, Streptococci, and Enterococcus faecalis in primary root canal infections. Journal of Endodontics $\mathbf{2 8}$, 168-72.

Sjögren U, Figdor D, Persson S, Sundqvist G (1997) Influence of infection at the time of root filling on the outcome of endodontic treatment of teeth with apical periodontitis. International Endodontic Journal 30, 297-306.

Sundqvist G (1976) Bacteriological Studies of Necrotic Dental Pulps. PhD Thesis (\#7). Umea, Sweden: University of Umea. 
Sundqvist G (1992) Ecology of the root canal flora. Journal of Endodontics 18, 427-30.

Sundqvist G, Figdor D, Persson S, Sjögren U (1998) Microbiologic analysis of teeth with failed endodontic treatment and the outcome of conservativere-treatment. Oral Surgery, Oral Medicine, Oral Pathology, Oral Radiology and Endodontics 85, 86-93.
Torabinejad M, Ung B, Kettering JD (1990) In vitro bacterial penetration of coronally unsealed endodontically treated teeth. Journal of Endodontics 16, 566-9.

Wu MK,Wesselink PR, BoersmaJ (1995) A1-year follow-up study on leakage of four root canal sealers at different thicknesses. International Endodontic Journal 28, 185-9. 